\title{
della Repubblica sociale italiana (Rsi). Intervista a Luigi Ganapini
}

ISSN: 2282-4979

DOI: 10.12977/ere-

view272

Questa intervista mira a mettere a confronto un autorevole punto di sintesi della storiografia sulla Rsi con le ipotesi interpretative proposte in questo dossier.

This interview aims to compare an authoritative synthesis point of historiography on Italian Social Republic with the interpretative hypothesis proposed in this dossier.

\section{Introduzione}

Luigi Ganapini è certamente uno dei principali studiosi della Repubblica sociale italiana. Alcuni dei suoi lavori costituiscono fondamentali riferimenti per i temi che poniamo in discussione in questo dossier. Tra questi, mi riferisco in particolare a Una città, la guerra. Milano 1939-1951 edito da Franco Angeli nel 1988, e a La Repubblica delle camicie nere, volume uscito da Garzanti nel 1999. Il primo libro è costituito da una raccolta di saggi che affronta - allora in termini pionieristici - aspetti sociali ed economici della vita nella città in guerra; e ritrae $\mathrm{i}$ tentativi delle autorità repubblicane milanesi (principalmente attraverso l'azione del podestà e capo provincia Piero Parini) di ottenere stabilità e consenso nel capoluogo lombardo, ossia in quello che era, a tutti gli effetti, il territorio più importante della Rsi.

Il secondo lavoro rappresenta invece un importante punto di arrivo per tutta quella storiografia che aveva finalmente cominciato a rinnovare ed ampliare pro- 
spettive e categorie di lettura di un soggetto, la Repubblica di Salò appunto, la cui interpretazione continuava a rimanere troppo spesso liminale al mutare delle contingenze politiche. A questo libro (e a quello di poco successivo di Dianella Gagliani) dobbiamo la prima, compiuta elaborazione di modelli e chiavi di lettura che restituiscono complessità e profondità, anche temporale, alle strutture della Repubblica e ai suoi aderenti; alle loro strategie di legittimazione volte a cercare pragmaticamente, nelle pieghe della società italiana modellata dal regime, ogni forma possibile di sostegno o accettazione.

A noi pare che la struttura amministrativa, contrariamente a quanto spesso si è ritenuto, sia stata uno dei piani su cui, di più e più a lungo, Salò ha cercato di giocare questa partita, ottenendovi anche qualche risultato che l'ha proiettata oltre la brevità della sua effettiva esistenza. Risultati da leggere però in relazione a persistenti continuità piuttosto che a drammatiche rotture; e a livello di una molteplicità di territori periferici, piuttosto che di un'unica dimensione centralistica. La video-intervista che presentiamo è stata realizzata proprio per cercare un confronto tra un autorevole punto di sintesi di quanto è stato fatto finora, e le ipotesi che stanno alla base di quanto qui si propone e degli approfondimenti che esse ci suggeriscono.

\section{Intervista}

1. Studiare Salò dal punto di vista amministrativo significa mettere a fuoco uomini e strutture con cui la Repubblica, fino all'ultimo, cercò di governare il proprio territorio. Ma il punto di vista più utile da adottare può essere quello dal centro?

a https://youtu.be/TJ1eZCGcsI4?rel=0

2. Ma se al centro della scena stanno le periferie, allora un punto di partenza fondamentale è dato dalla figura del capo-provincia. Qual è il suo significato per la Repubblica sociale?

O https://youtu.be/m3jbYV8s2k4?rel=0

3. In una situazione così frammentata, i capi-provincia devono giocare un ruolo complesso, su cui però prevalgono ancora interpretazioni semplificatorie, come la secca bipartizione tra estremisti e moderati.

a https://youtu.be/3DVHXHkXgk8?rel=0 
4. Dagli uomini ai tempi. È possibile una periodizzazione che non sia legata solo alla guerra e alla violenza, ma anche alle vicende interne e alle strutture meno esplicitamente politiche della Rsi?

$\boldsymbol{O}$ https://youtu.be/rKVqajdI5Yk?rel=0

5. Cominciamo allora proprio dal principale dei territori della Rsi, Milano. Qual è il significato della città per la Repubblica e quale la sua periodizzazione?

Q $\underline{\text { https://youtu.be/NmfTUm1MOvw?rel=0 }}$

6. Nell'inverno 1944-1945 c'è la definitiva delegittimazione della Repubblica, sul filo della violenza, ma anche dell'ormai evidente fallimento amministrativo. Quali ne sono i segni a Milano?

A https://youtu.be/-callFO2wWU?rel=0

7. E gli altri territori della Repubblica? Come possiamo arrivare a identificarli?

Q 1 https://youtu.be/4OFYkJyOVY?rel=0

8. Su quali punti dello Stato fascista la Rsi può meglio fondare la sua partita amministrativa? $\mathrm{E}$ che parte vi ha la continuità?

A https://youtu.be/fdni20Q0Pj0?rel=0

9. Possiamo allora dire che la Rsi ha avuto a lungo una progettualità amministrativa, che era una prospettiva di continuità e consenso, certo molto fragile e tuttavia complessa da definire nei suoi uomini e nelle sue modalità?

A https://youtu.be/OwZIbsFFClg?rel=0

Riprese: Igor Pizzirusso Montaggio: Liviana Davì

\section{Bibliografia}

Ganapini L. 1988, Una città, la guerra. Milano 1939-1951, Milano: Franco Angeli

Ganapini L. 1999, La Repubblica delle camicie nere, Milano: Garzanti

Ganapini L. 2012, Voci dalla guerra civile. Italiani nel 1943-1945, Bologna: il Mulino 